\title{
On an Identification Problem on the Determination of the Parameters of the Dynamic System
}

\author{
Dossan Baigereyev, ${ }^{1}$ Nevazi Ismailov, ${ }^{2,3}$ Yusif Gasimov, ${ }^{2,4}$ and Atif Namazov ${ }^{2}$ \\ ${ }^{1}$ Higher Mathematics Department, D. Serikbayev East Kazakhstan State Technical University, Oskemen, Kazakhstan \\ ${ }^{2}$ Institute of Applied Mathematics, Baku State University, Z. Khalilov 23, AZ1148 Baku, Azerbaijan \\ ${ }^{3}$ Institute of Information Technologies of ANAS, B. Vahabzade 9, AZ1141 Baku, Azerbaijan \\ ${ }^{4}$ Institute of Mathematics and Mechanics of ANAS, Baku, Azerbaijan
}

Correspondence should be addressed to Dossan Baigereyev; dbaigereyev@gmail.com

Received 13 February 2015; Accepted 15 June 2015

Academic Editor: Manuel Ruiz Galán

Copyright (C) 2015 Dossan Baigereyev et al. This is an open access article distributed under the Creative Commons Attribution License, which permits unrestricted use, distribution, and reproduction in any medium, provided the original work is properly cited.

An inverse problem is considered for the determination of the parameters, involved in the right-hand side of the system of nonlinear ordinary differential equations by given initial and final conditions. The solution of the problem is reduced to the minimization of the quadratic functional, which indeed is a deviation of the value of the solution from the given values at the end points. Using the quasilinearization method a calculation method is proposed to the solution of the considered problem. The application of this method is demonstrated on the example of the determination of the hydraulic resistance in the tubes.

\section{Introduction}

As is known, different classes of inverse and identification problems play an important role in the solition of many applied problems from physics, hydrodynamics, and industry [1-5]. There exist various methods to solve these problems, as well as optimization methods [6-10]. One of the principle steps of these methods is a choice of suitable functional. Since many applied problems are described by the nonlinear systems, the choice of such functional and further solution of the corresponding optimization problem is problematic [2]. These difficulties may be avoid, for example, by the iterational quasilinearization method, convergence of which is in detail studied in $[11,12]$.

In the present work a multidimensional identification problem is considered for the determination of the parameters involving the right-hand side of the system of nonlinear differential equations by given initial and final conditions. Solution of the problem is reduced to the optimization problem, in which the functional under minimization is constructed as a quadratic deviation of the solition of the system from the given data at the end points. Since solution of the problem in the stated nonlinear formulation presents certain difficulties $[13,14]$, the considered problem is reduced to the linear case with respect to phase coordinates and vector of parameters by the help of quasilinearization method. A quadratic functional is constructed and an expression for its gradient is derived. Using Gram-Schmidt orthogonalization method a calculation algorithm is proposed, which allows one to define sought parameters. This algorithm is applied to the example, describing the flow in the pipes.

\section{Problem Statement}

Let the movement of the object be described by the system of differential equations:

$$
\dot{y}(t)=f(y(t), \alpha),
$$

where $y$ is $n$-dimensional phase vector, $f$ is $n$-dimensional differentiable function continuous in the interval $(0, T)$, and $\alpha$ is $m$-dimensional constant vector to be found.

Let the following initial conditions be given:

$$
y^{i}(0)=y_{0}^{i}, \quad \overline{i=1, N}
$$


where $N n, m$ are given natural numbers and $t$ is an independent variable $y_{0}^{i}, i=\overline{1, N}$ given $n$-dimensional vector. The problem consists in the finding of the vector $\alpha$, by which the solution of the Cauchy problem (1)-(2) in the point $T$ satisfies the given condition

$$
y^{i}(T)=y_{T}^{i}, \quad \overline{i=1, N} .
$$

Such problems are often met in applications $[1,3,6,15]$, when initial data (2) are given and final ones are statistically measured. In these cases it is required to find the vector $\alpha$ such that the solution of the problem by initial data (2) is maximally close to the measured data at the end points. As an example the problem in oil-gas production may be shown when it needs to define the coefficient of the hydraulic resistance.

\section{Solution Method}

Since the function $f(y, \alpha)$ is nonlinear, to solve the problem (1)-(3) it is expedient to use any numerical method, as well as quasilinearization method $[11,13]$. So in the first step we linearize the problem (1)-(3). For this purpose some nominal trajectory $y^{0}(t)$ and parameter $\alpha^{0}$ are chosen and it is assumed that $k$ th iteration is already hold. If we linearize (1) with respect to these data we obtain

$$
\begin{aligned}
\dot{y}^{k}= & \frac{\partial f\left(y^{k-1}, \alpha^{k-1}\right)}{\partial y} y^{k}+\frac{\partial f\left(y^{k-1}, \alpha^{k-1}\right)}{\partial \alpha} \alpha^{k} \\
& +f\left(y^{k-1}, \alpha^{k-1}\right)-\frac{\partial f\left(y^{k-1}, \alpha^{k-1}\right)}{\partial y} y^{k-1} \\
& -\frac{\partial f\left(y^{k-1}, \alpha^{k-1}\right)}{\partial \alpha} \alpha^{k-1} .
\end{aligned}
$$

After integration of the linear differential equation (4) with condition (2) we get the representation [16]

$$
\begin{aligned}
y^{k}(t)= & \Phi^{k-1}\left(t, t_{0}\right) \cdot y^{k}\left(t_{0}\right)+\Phi_{1}^{k-1}\left(t, t_{0}\right) \cdot \alpha^{k} \\
& +\Phi_{2}^{k-1}\left(t, t_{0}\right),
\end{aligned}
$$

where $\Phi^{k-1}\left(t, t_{0}\right)$ is a fundamental matrix of the system of homogeneous equations

$$
\dot{y}^{k}(t)=\left.\frac{\partial f\left(y^{k-1}, \alpha^{k-1}\right)}{\partial y}\right|_{\substack{y=y^{k-1} \\ \alpha=\alpha^{k-1}}} \cdot y^{k}\left(t_{0}\right)
$$

and the matrices $\Phi_{1}^{k-1}\left(t, t_{0}\right), \Phi_{2}^{k-1}\left(t, t_{0}\right)$ are defined as in [16]

$$
\begin{aligned}
& \Phi_{1}^{k-1}\left(t, t_{0}\right)=\int_{t_{0}}^{t} \Phi(t, \tau) \frac{\partial f\left(y^{k-1}, \alpha^{k-1}\right)}{\partial \alpha} d \tau, \\
& \Phi_{2}^{k-1}\left(t, t_{0}\right)=\int_{t_{0}}^{t} \Phi(t, \tau)\left[f\left(y^{k-1}, \alpha^{k-1}\right)\right. \\
& \left.\quad-\frac{\partial f\left(y^{k-1}, \alpha^{k-1}\right)}{\partial y} y^{k-1}-\frac{\partial f\left(y^{k-1}, \alpha^{k-1}\right)}{\partial \alpha} \alpha^{k-1}\right] d \tau .
\end{aligned}
$$

To provide that the solution $y^{i}(T)$ of the linearized differential equation (4) with initial data (2) coincides with the values of the measurements $y_{T}^{i}=y_{T i}$ in the point $T$ we construct the following quadratic functional in the $k$ th iteration:

$$
I_{k}=\frac{1}{2} \sum_{i=1}^{N}\left[y_{i}^{k}(T, \alpha)-y_{T i}^{k}\right]^{T} A_{i}^{k}\left[y_{i}^{k}(T, \alpha)-y_{T i}^{k}\right],
$$

where the sign $T$ stands for transpore, $A^{k}$ is a constant symmetre $m \times n$-dimensional weight matrix, that is chosen in each iteration, considering the specifics of the concrete problem; $y_{T i}^{k}$ is $n \times 1$-dimensional vector of observation; $y_{i}^{k}$ is $n \times 1$-dimensional vector defined by (5). Then the solution of the stated problem is reduced to the problem: Find a constant vector $\alpha$, by which the solution of (1) with conditions (2) minimizes the functional (8).

Various algorithms exist for the minimization of the functional (8). However in the solution of the concrete problem, as well as problem arising in the oil production, these algorithms met some difficulties [14] (e.g., to reach the necessary accuracy and speed of convergence). Therefore in [6] the use of Gram-Schmidt orthogonalization method is proposed.

\section{Algorithm for the Minimization of the Functional (8)}

Here we consider the minimization of the functional (8) by the help of the relation (4) with conditions (2). Putting $y^{k}(T)$ from (5) into (8) we get

$$
\begin{aligned}
I_{k} & =\frac{1}{2} \sum_{i=1}^{N}\left[\Phi_{i}^{k-1}\left(T, t_{0}\right) y_{i}^{k}\left(t_{0}\right)+\Phi_{1 i}^{k-1}\left(T, t_{0}\right) \cdot \alpha\right. \\
& \left.+\Phi_{2 i}^{k-1}\left(T, t_{0}\right)-y_{T i}\right]^{T} A_{i}^{k}\left[\Phi_{i}^{k-1}\left(T, t_{0}\right) y_{i}^{k}\left(t_{0}\right)\right. \\
& \left.+\Phi_{1 i}^{k-1}\left(T, t_{0}\right) \cdot \alpha+\Phi_{2 i}^{k-1}\left(T, t_{0}\right)-y_{T i}\right]=\sum_{j=1}^{16} I_{k}^{j} .
\end{aligned}
$$

Considering the symmetricity of the matrix $A^{k}$ the relation (9) may be written as

$$
\begin{aligned}
& I_{k}^{1} \stackrel{\text { def }}{=} \frac{1}{2} \\
& \cdot \sum_{i=1}^{N}\left[\Phi_{i}^{k-1}\left(T, t_{0}\right) y_{i}^{k}\left(t_{0}\right)\right]^{T} A_{i}^{k} \Phi_{i}^{k-1}\left(T, t_{0}\right) y_{i}^{k}\left(t_{0}\right), \\
& I_{k}^{2} \stackrel{\text { def }}{=} \frac{1}{2} \sum_{i=1}^{N}\left[\Phi_{i}^{k-1}\left(T, t_{0}\right) y_{i}^{k}\left(t_{0}\right)\right]^{T} A_{i}^{k} \Phi_{1 i}^{k-1}\left(T, t_{0}\right) \cdot \alpha, \\
& I_{k}^{3} \stackrel{\text { def }}{=} \frac{1}{2} \sum_{i=1}^{N}\left[\Phi_{i}^{k-1}\left(T, t_{0}\right) y_{i}^{k}\left(t_{0}\right)\right]^{T} A_{i}^{k} \Phi_{2 i}^{k-1}\left(T, t_{0}\right), \\
& I_{k}^{4} \stackrel{\text { def }}{=}-\frac{1}{2} \sum_{i=1}^{N}\left[\Phi_{i}^{k-1}\left(T, t_{0}\right) y_{i}^{k}\left(t_{0}\right)\right]^{T} A_{i}^{k} y_{T i}^{k},
\end{aligned}
$$




$$
\begin{aligned}
& I_{k}^{5} \stackrel{\text { def }}{=} \frac{1}{2} \sum_{i=1}^{N}\left[\Phi_{1 i}^{k-1}\left(T, t_{0}\right) \cdot \alpha\right]^{T} A_{i}^{k} \Phi_{i}^{k-1}\left(T, t_{0}\right) y_{i}^{k}\left(t_{0}\right), \\
& I_{k}^{6} \stackrel{\text { def }}{=} \frac{1}{2} \sum_{i=1}^{N}\left[\Phi_{1 i}^{k-1}\left(T, t_{0}\right) \cdot \alpha\right]^{T} A_{i}^{k} \Phi_{1 i}^{k-1}\left(T, t_{0}\right) \cdot \alpha, \\
& I_{k}^{7} \stackrel{\text { def }}{=} \frac{1}{2} \sum_{i=1}^{N}\left[\Phi_{1 i}^{k-1}\left(T, t_{0}\right) \cdot \alpha\right]^{T} A_{i}^{k} \Phi_{2 i}^{k-1}\left(T, t_{0}\right), \\
& I_{k}^{8} \stackrel{\text { def }}{=}-\frac{1}{2} \sum_{i=1}^{N}\left[\Phi_{1 i}^{k-1}\left(T, t_{0}\right) \cdot \alpha\right]^{T} A_{i}^{k} y_{T i}^{k}, \\
& I_{k}^{9} \stackrel{\text { def }}{=} \frac{1}{2} \sum_{i=1}^{N}\left[\Phi_{2 i}^{k-1}\left(T, t_{0}\right)\right]^{T} A_{i}^{k} \Phi_{i}^{k-1}\left(T, t_{0}\right) y_{i}^{k}\left(t_{0}\right), \\
& I_{k}^{15} \stackrel{\text { def }}{=}-\frac{1}{=} \sum_{i=1}^{N}\left[y_{T i}^{k}\right]^{T} A_{i}^{k} y_{T i}^{k} \\
& I_{k}^{14} \stackrel{1}{2} \sum_{i=1}^{N}\left[\Phi_{2 i}^{k-1}\left(T, t_{0}\right)\right]^{T} A_{i}^{k} \Phi_{1 i}^{k-1}\left(T, t_{0}\right) \cdot \alpha, \\
& \left.I_{k}^{13} \stackrel{\frac{\text { def }}{=}}{=}-\frac{1}{2} \sum_{i=1}^{N}\left[y_{T i}^{k}\right]_{i=1}^{k}\right]_{i}^{T} A_{i}^{k} \Phi_{i}^{k} \Phi_{2 i}^{k-1}\left(T, y_{0}^{k}\right) y_{i}^{k}\left(t_{0}\right), \\
& I_{k}^{11} \stackrel{\text { def }}{=} \frac{1}{2} \sum_{i=1}^{N}\left[\Phi_{2 i}^{k-1}\left(T, t_{0}\right)\right]^{T} A_{i}^{k} \Phi_{2 i}^{k-1}\left(T, t_{0}\right), \\
& \stackrel{1}{k} \Phi_{1 i}^{k-1}\left(T, t_{0}\right) \cdot \alpha, \\
& \left.I_{2 i}^{k-1}\left(T, t_{0}\right)\right]^{T} A_{i}^{k} y_{T i}^{k},
\end{aligned}
$$

and gradient of the functional (9) has a form

$$
\frac{\partial I_{k}}{\partial \alpha}=\sum_{j=1}^{16} \frac{\partial I_{k}^{j}}{\partial \alpha}
$$

Since the terms $I_{k}^{1}, I_{k}^{3}, I_{k}^{4}, I_{k}^{9}, I_{k}^{11}, I_{k}^{12}, I_{k}^{13}, I_{k}^{15}, I_{k}^{16}$ do not depend on the parameter $\alpha$, we have

$$
\begin{aligned}
\frac{\partial I_{k}^{1}}{\partial \alpha} & =\frac{\partial I_{k}^{3}}{\partial \alpha}=\frac{\partial I_{k}^{4}}{\partial \alpha}=\frac{\partial I_{k}^{9}}{\partial \alpha}=\frac{\partial I_{k}^{11}}{\partial \alpha}=\frac{\partial I_{k}^{12}}{\partial \alpha}=\frac{\partial I_{k}^{13}}{\partial \alpha} \\
& =\frac{\partial I_{k}^{15}}{\partial \alpha}=\frac{\partial I_{k}^{16}}{\partial \alpha}=0 .
\end{aligned}
$$

Based on the formulas

$$
\begin{array}{r}
\frac{\partial x^{T} a}{\partial x}=\frac{\partial a^{T} x}{\partial x}=a, \\
\frac{\partial x^{T} B x}{\partial x}=\left(B+B^{T}\right) x
\end{array}
$$

from $[17,18]$, for the gradients of $I_{k}^{2}, I_{k}^{5}, I_{k}^{6}, I_{k}^{7}, I_{k}^{8}, I_{k}^{10}, I_{k}^{14}$, we get the formulas

$$
\begin{aligned}
& \frac{I_{k}^{2}}{\partial \alpha}=\left(\left[\Phi_{i}^{k-1}\left(T, t_{0}\right) y_{i}^{k}\left(t_{0}\right)\right]^{T} A_{i}^{k} \Phi_{1 i}^{k-1}\left(T, t_{0}\right)\right)^{T} \\
& =\Phi_{1 i}^{k-1}\left(T, t_{0}\right) A_{i}^{k^{T}} \Phi_{i}^{k-1}\left(T, t_{0}\right) y_{i}^{k}\left(t_{0}\right), \\
& \frac{I_{k}^{5}}{\partial \alpha}=\frac{\alpha^{T} \Phi_{1 i}^{k-1^{T}}\left(T, t_{0}\right) A_{i}^{k} \Phi_{i}^{k-1}\left(T, t_{0}\right) y_{i}^{k}\left(t_{0}\right)}{\partial \alpha} \\
& =\Phi_{1 i}^{k-1^{T}}\left(T, t_{0}\right) A_{i}^{k} \Phi_{i}^{k-1}\left(T, t_{0}\right) y_{i}^{k}\left(t_{0}\right), \\
& \frac{I_{k}^{6}}{\partial \alpha}=\frac{\alpha^{T} \Phi_{1 i}^{k-1}\left(T, t_{0}\right) A_{i}^{k} \Phi_{1 i}^{k-1}\left(T, t_{0}\right) \alpha}{\partial \alpha} \\
& =\left[\Phi_{1 i}^{k-1^{T}}\left(T, t_{0}\right) A_{i}^{k} \Phi_{1 i}^{k-1}\left(T, t_{0}\right)\right. \\
& \left.+\Phi_{1 i}^{k-1^{T}}\left(T, t_{0}\right) A_{i}^{k^{T}} \Phi_{1 i}^{k-1}\left(T, t_{0}\right)\right] \alpha \\
& =2 \Phi_{1 i}^{k-1^{T}}\left(T, t_{0}\right) A_{i}^{k} \Phi_{1 i}^{k-1}\left(T, t_{0}\right) \alpha, \\
& \frac{I_{k}^{7}}{\partial \alpha}=\frac{\alpha^{T} \Phi_{1 i}^{k-1^{T}}\left(T, t_{0}\right) A_{i}^{k} \Phi_{2 i}^{k-1}\left(T, t_{0}\right)}{\partial \alpha}=\Phi_{1 i}^{k-1^{T}}\left(T, t_{0}\right) \\
& \cdot A_{i}^{k} \Phi_{2 i}^{k-1}\left(T, t_{0}\right)
\end{aligned}
$$

$$
\begin{aligned}
\frac{I_{k}^{8}}{\partial \alpha} & =-\frac{\left[\Phi_{1 i}^{k-1}\left(T, t_{0}\right) \alpha\right]^{T} A_{i}^{k} y_{T i}^{k}}{\partial \alpha} \\
& =-\frac{\alpha^{T} \Phi_{1 i}^{k-1}\left(T, t_{0}\right) A_{i}^{k} y_{T i}^{k}}{\partial \alpha}=-\Phi_{1 i}^{k-1}\left(T, t_{0}\right) \\
& \cdot A_{i}^{k} y_{T i}^{k},
\end{aligned}
$$$$
\frac{I_{k}^{10}}{\partial \alpha}=\Phi_{1 i}^{k-1}\left(T, t_{0}\right) A_{i}^{k^{T}} \Phi_{2 i}^{k-1}\left(T, t_{0}\right)
$$$$
\frac{I_{k}^{14}}{\partial \alpha}=-\frac{\left[y_{T i}^{k}\right]^{T} A_{i}^{k} \Phi_{1 i}^{k-1}\left(T, t_{0}\right) \alpha}{\partial \alpha}=-\Phi_{1 i}^{k-1^{T}}\left(T, t_{0}\right)
$$$$
\cdot A_{i}^{k^{T}} y_{T i}^{k}
$$ 
Finally if we consider these results, then the gradient of the functional (9) will be defined by the formula

$$
\begin{aligned}
\frac{\partial I_{k}}{\partial \alpha} & =\frac{1}{2} \sum_{i=1}^{N}\left(\frac{I_{k}^{2}}{\partial \alpha}+\frac{I_{k}^{5}}{\partial \alpha}+\frac{I_{k}^{6}}{\partial \alpha}+\frac{I_{k}^{7}}{\partial \alpha}+\frac{I_{k}^{8}}{\partial \alpha}+\frac{I_{k}^{10}}{\partial \alpha}\right. \\
& \left.+\frac{I_{k}^{14}}{\partial \alpha}\right)=\frac{1}{2} \\
& +\sum_{i=1}^{N}\left(\Phi_{1 i}^{k-1}\left(T, t_{0}\right) A_{i}^{k^{T}} \Phi_{i}^{k-1}\left(T, t_{0}\right) y_{i}^{k}\left(t_{0}\right)\right. \\
& +\Phi_{1 i}^{k-1}\left(T, t_{0}\right) A_{i}^{k} \Phi_{i}^{k-1}\left(T, t_{0}\right) y_{i}^{k}\left(t_{0}\right) \\
& +2 \Phi_{1 i}^{k-1}\left(T, t_{0}\right) A_{i}^{k} \Phi_{1 i}^{k-1}\left(T, t_{0}\right) \alpha \\
& +\Phi_{1 i}^{k-1}\left(T, t_{0}\right) A_{i}^{k} \Phi_{2 i}^{k-1}\left(T, t_{0}\right) \\
& -\Phi_{1 i}^{k-1}\left(T, t_{0}\right) A_{i}^{k} y_{T i}^{k} \\
+ & \Phi_{1 i}^{k-1}\left(T, t_{0}\right) A_{i}^{k^{T}} \Phi_{2 i}^{k-1}\left(T, t_{0}\right) \\
& +2 \Phi_{1 i}^{k-1}\left(T, t_{0}\right) A_{i}^{k} \Phi_{2 i}^{k-1}\left(T, t_{0}\right) \\
& \left.+\Phi_{1 i}^{k-1}\left(T, t_{0}\right) A_{i}^{k^{T}} y_{T i}^{k}\right)=\frac{1}{2} \\
& +\sum_{i=1}^{N}\left(2 \Phi_{1 i}^{k-1}\left(T, t_{0}\right) A_{i}^{k} y_{T i}^{k}\right)=0 . \\
& \\
+ &
\end{aligned}
$$

Then, for the gradient of the functional $I_{k}$ relatively to the parameter $\alpha$ we get the expression

$$
\begin{aligned}
\frac{\partial I_{k}}{\partial \alpha} & =\sum_{i=1}^{N}\left[\Phi_{1 i}^{k-1^{T}}\left(T, t_{0}\right) A_{i}^{k} \Phi_{1 i}^{k-1}\left(T, t_{0}\right)\right] \alpha \\
& -\sum_{i=1}^{N}\left[\Phi_{1 i}^{k-1^{T}}\left(T, t_{0}\right) A_{i}^{k} y_{T i}^{k}\right. \\
& -\Phi_{1 i}^{k-1^{T}}\left(T, t_{0}\right) A_{i}^{k^{T}} \Phi_{i}^{k-1}\left(T, t_{0}\right) y_{i}^{k}\left(t_{0}\right) \\
& \left.-\Phi_{1 i}^{k-1}\left(T, t_{0}\right) A_{i}^{k} \Phi_{2 i}^{k-1}\left(T, t_{0}\right)\right] .
\end{aligned}
$$

Taking equal to zero the expression (16) we get

$$
\begin{gathered}
\sum_{i=1}^{N}\left[\Phi_{1 i}^{k-1^{T}}\left(T, t_{0}\right) A_{i}^{k} \Phi_{1 i}^{k-1}\left(T, t_{0}\right)\right] \alpha \\
=\sum_{i=1}^{N}\left[\Phi_{1 i}^{k-1^{T}}\left(T, t_{0}\right) A_{i}^{k} y_{T i}^{k}\right.
\end{gathered}
$$

$$
\begin{aligned}
& -\Phi_{1 i}^{k-1^{T}}\left(T, t_{0}\right) A_{i}^{k^{T}} \Phi_{i}^{k-1}\left(T, t_{0}\right) y_{i}^{k}\left(t_{0}\right) \\
& \left.-\Phi_{1 i}^{k-1}\left(T, t_{0}\right) A_{i}^{k} \Phi_{2 i}^{k-1}\left(T, t_{0}\right)\right] .
\end{aligned}
$$

Solution of (17) with respect to $\alpha$ gives

$$
\begin{aligned}
\alpha= & {\left[\sum_{i=1}^{N} \Phi_{1 i}^{k-1^{T}}\left(T, t_{0}\right) A_{i}^{k} \Phi_{1 i}^{k-1}\left(T, t_{0}\right)\right]^{-1} } \\
& \cdot \sum_{i=1}^{N}\left[\Phi_{1 i}^{k-1}\left(T, t_{0}\right) A_{i}^{k} y_{T i}^{k}\right. \\
& -\Phi_{1 i}^{k-1^{T}}\left(T, t_{0}\right) A_{i}^{k^{T}} \Phi_{i}^{k-1}\left(T, t_{0}\right) y_{i}^{k}\left(t_{0}\right) \\
& \left.-\Phi_{1 i}^{k-1}\left(T, t_{0}\right) A_{i}^{k} \Phi_{2 i}^{k-1}\left(T, t_{0}\right)\right]
\end{aligned}
$$

where it is assumed that $\left[\sum_{i=1}^{N} \Phi_{1 i}^{k-1^{T}}\left(T, t_{0}\right) A_{i}^{k} \Phi_{1 i}^{k-1}\left(T, t_{0}\right)\right]^{-1}$ exists.

Value of the parameter $\alpha$, defined by (18) is a solution of the multiparameter optimization problem for the functional (9) that gives minimum to the cost functional.

Considering the above the following algorithm may be proposed to the solution of the identification problem (1), (2).

Algorithm 1. (1) Construct the function $f(x)$ from (1), initial and final data $y_{0}^{i}$ and $y_{T}^{i}(i=1, N)$ from (2) and (3) correspondingly.

(2) Calculate the derivatives $\partial f\left(y^{k-1}, \alpha^{k-1}\right) / \partial y, \partial f\left(y^{k-1}\right.$, $\left.\alpha^{k-1}\right) / \partial \alpha$ taking as initial approaches $y_{i}$ and $\alpha_{i}$.

(3) Calculate the fundamental matrix $\Phi^{k-1}\left(t, t_{0}\right)$ from (6); reconstruct $\Phi_{1}^{k-1}\left(t, t_{0}\right), \Phi_{2}^{k-1}\left(t, t_{0}\right)$ from (7) and functional $I_{k}$ from (8).

(4) Solving the system of algebraic equations (14) relatively $\alpha$ find the value of the $m$-dimensional vector $\alpha^{k}$ in the $k$ th iteration.

(5) Check the condition

$$
\left|\frac{\partial I_{k}}{\partial \alpha}\right|<\varepsilon
$$

where $\varepsilon$ is a given small enough number. If the condition (16) is satisfied the process stops; otherwise go to Step (2). The convergence of this algorithm may be proved similar to $[13,14]$.

Now we discuss the realization of this algorithm.

\section{Calculational Algorithm}

As one can see, in the realization of the above algorithm the main step is a calculation of the fundamental matrix $\Phi^{k-1}\left(t, t_{0}\right)$ and the matrices $\Phi_{1}^{k-1}\left(t, t_{0}\right), \Phi_{2}^{k-1}\left(t, t_{0}\right)$. Note that, as is mentioned in [16], construction of these matrices is an enough difficult procedure. So for simplicity we try (4) and find the corresponding derivatives by using the Euler method. 
Really $\partial f(y(t), \alpha) / \partial y$ and $\partial f(y(t), \alpha) / \partial \alpha$ everywhere are replaced by $\tilde{f}(y)=(f(y+\delta, \alpha)-f(y, \alpha)) / \delta$ and $\tilde{\tilde{f}}(\alpha)=$ $\left(f\left(y, \alpha+\delta_{1}\right)-f(y, \alpha)\right) / \delta_{1}$ correspondingly, where $\delta$ and $\delta_{1}$ are small enough numbers.

To calculate the fundamental matrix $\Phi^{k-1}\left(t, t_{0}\right)$ and the matrices $\Phi_{1}^{k-1}\left(t, t_{0}\right), \Phi_{2}^{k-1}\left(t, t_{0}\right)$ it is proper to replace (4) by the following discrete one:

$$
\begin{aligned}
y^{k}\left(t_{2 N}\right) & \\
= & \left(\prod_{i_{1}=2 N-1}^{j}\left(E+\delta \tilde{\widetilde{f}}\left(y^{k-1}\left(t_{i_{1}}\right)\right)\right)\right) y^{k}\left(t_{N+1}\right) \\
& +\Phi_{1}^{k-1}\left(t, t_{0}\right) \alpha+\Phi_{2}^{k-1}\left(t, t_{0}\right),
\end{aligned}
$$

where

$$
\begin{aligned}
& \Phi_{i}^{k-1}\left(t_{2 N}, t_{0}\right)=\prod_{i_{1}=2 N-1}^{j}\left(E+\delta \tilde{\tilde{f}}\left(y^{k-1}\left(t_{i}\right)\right)\right), \\
& \Phi_{1 i}^{k-1}\left(t_{2 N}, t_{0}\right) \\
& =\left(\sum_{j=N+2}^{2 N-1}\left(\prod_{i_{1}=2 N-1}^{j}\left(E+\delta \cdot \tilde{\tilde{f}}\left(y^{k-1}\left(t_{i}\right)\right)\right)\right)\right. \\
& \left.\cdot \delta \tilde{\tilde{f}}\left(\alpha_{j-1}^{k-1}\right)\right)+\delta \cdot \tilde{\tilde{f}}\left(\alpha_{2 N-1}^{k-1}\right), \\
& \Phi_{2 i}^{k-1}\left(t_{2 N}, t_{0}\right) \\
& =\left(\sum_{j=N+2}^{2 N-1}\left(\prod_{i_{1}=2 N-1}^{j}\left(E+\delta \cdot \tilde{\tilde{f}}\left(y^{k-1}\left(t_{i}\right)\right)\right)\right) \delta\right. \\
& \cdot\left(\tilde{f}\left(y^{k-1}\left(t_{j-1}\right), \alpha^{k-1}\right)\right. \\
& \left.\left.\quad-\tilde{\tilde{f}}\left(y^{k-1}\left(t_{j-1}\right)\right) y^{k-1}\left(t_{j-1}\right)-\tilde{\tilde{f}}\left(\alpha^{k-1}\right) \alpha^{k-1}\right)\right) \\
& \quad+\delta \cdot\left(\tilde{f}\left(y^{k-1}\left(t_{2 N-1}\right), \alpha^{k-1}\right)-\tilde{\tilde{f}}\left(y^{k-1}\left(t_{2 N-1}\right)\right)\right. \\
& \left.\cdot y^{k-1}\left(t_{2 N-1}\right)-\tilde{\tilde{f}}\left(\alpha^{k-1}\right) \alpha^{k-1}\right) .
\end{aligned}
$$

$E$ is unit matrix of proper dimension.

Then from (20) we get that $\Phi^{k-1}\left(t, t_{0}\right)$ is a fundamental matrix for the system of homogeneous equations:

$$
y^{k}\left(t_{i+1}\right)=\left.(E+\delta \tilde{f}(y))\right|_{\substack{y=y^{k-1} \\ \alpha=\alpha^{k-1}}} \cdot y^{k}\left(t_{i}\right) .
$$

Therefore, similar to the nongradient methods we propose an algorithm, based on the orthogonalization of gradient directions using the Gram-Schmidt procedure.
Step 1. Using Gram-Schmidt orthogonalization, vectors $\omega_{i}^{j}$, $i=j, j+1, \ldots, n, j=2,3, \ldots, n^{2}$ are calculated and the set of vectors

$$
\left(\frac{\partial f\left(\lambda_{c}^{1}\right)}{\partial \lambda_{c}^{1}}, \frac{\partial f\left(\lambda_{c}^{2}\right)}{\partial \lambda_{c}^{2}}, \ldots, \frac{\partial f\left(\lambda_{c}^{i}\right)}{\partial \lambda_{c}^{i}}, \omega_{i+1}^{j}, \ldots, \omega_{n}^{j}\right)
$$

is found which form an orthogonal basis in $R^{n}$.

If we apply the orthogonalization algorithm, then a linearly independent system $a_{1}, a_{2}, \ldots, a_{k}$ should be formed, that is, orthogonal system $b_{1}, b_{2}, \ldots, b_{k}$ and each vector $b_{i}$ should be linearly expressed through $a_{1}, a_{2}, \ldots, a_{i}$. Here $a_{i}$ and $b_{i}$ are upper triangular matrices. Thus it is possible to ensure that the systems $\left\{b_{i}\right\}$ were orthonormal, where the diagonal elements of the transition matrix are positive; by these conditions the system $\left\{b_{i}\right\}$ and the transition matrix are uniquely determined.

The algorithm considers $b_{1}=a_{1}$, if the vectors, $b_{1}, b_{2}, \ldots, b_{i-1}$, are constructed. Then

$$
b_{i}=a_{i}-\sum_{j=1}^{i-1} \frac{\left\langle a_{i}, b_{j}\right\rangle}{\left\langle b_{i}, b_{j}\right\rangle} b_{j},
$$

where \langle\rangle is the sign of the scalar product of vectors.

Step 2. For the orthogonalization of the gradient directions we compute $v_{i}^{j}$ in the form

$$
v_{i}^{j}=\frac{f\left(\lambda_{c}+\delta \omega_{i}^{j}\right)-f\left(\lambda_{c}-\delta \omega_{i}^{j}\right)}{2 \delta}, \frac{}{i=j, j+1, \ldots, n .}
$$

Here $\delta>0$ is any small parameter.

Step 3. The orthogonal gradient directions are chosen in the form

$$
l_{j}=\sum_{i=j}^{n} v_{i}^{j} \omega_{i}^{j}
$$

Replacing $\nabla f\left(\lambda_{c}^{(k)}\right)$ by $l_{j}$ in (17) the nongradient iterative minimization procedure will be

$$
\lambda_{c}^{(k+1)}=\lambda_{c}^{(k)}-\chi^{*(k)} l_{k}
$$

where $\chi^{*(k)}$ is a scalar, which is determined by golden section method.

Now we apply the above proposed technique to the example of 15 production by gas-lift method.

Example 2. It is known that nonstable motion of gas in tubes and gas liquid mixture (GLM) in vertical tubes, that is, in the lift pipe of the gas-lift well with constant across profile, is 


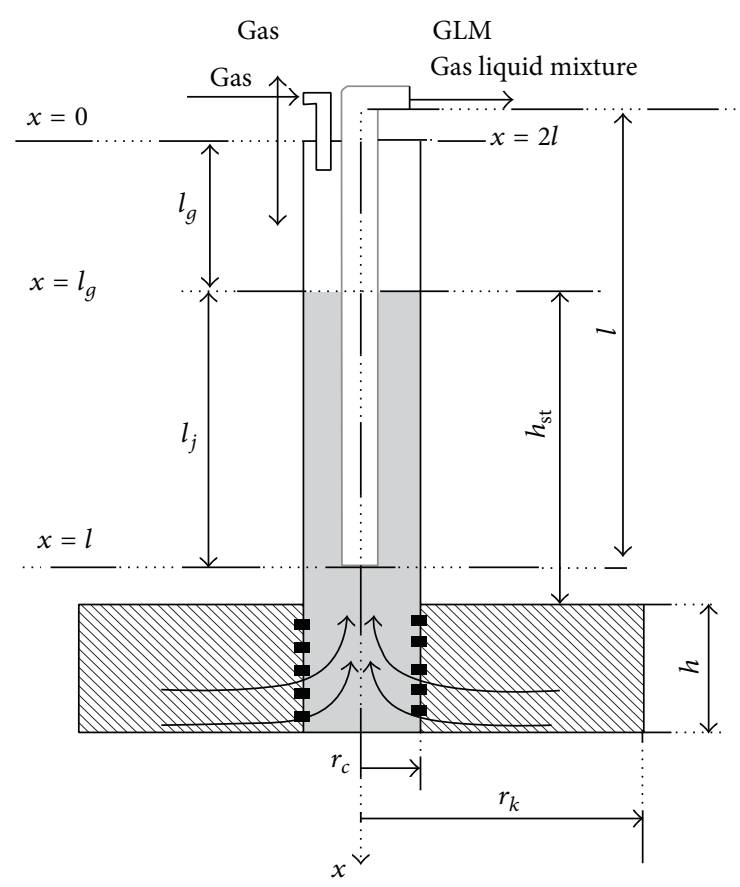

FIgURe 1

described by the following system of linear partial differential equations of hyperbolic type (see Figure 1):

$$
\begin{aligned}
& -\frac{\partial P}{\partial x}=\frac{\partial\left(\rho \omega_{c}\right)}{\partial t}+2 \alpha \rho \omega_{c}, \\
& -\frac{\partial P}{\partial t}=c^{2} \frac{\partial\left(\rho \omega_{c}\right)}{\partial x},
\end{aligned}
$$

where $\rho=P(x, t), \omega_{c}=\omega_{c}(x, t)$, is an additional pressure on its stationary value and averaged over across section speed of motion of GLM; $t, x$ : time and coordinate; $c$ : speed of sound in gas and GLM; $\rho$ : gas, oil, and GLM in correspondence with coordinates; $2 \alpha=g / \omega_{c}+\lambda_{c} \omega_{c} / 2 D ; g, \lambda_{c}$ : free fall accel ...; $D$ : interval effective diameter of the tube [19].

The partial differential equation of gas and GLM motion by are averaging over time $t$ may be reduced to the following ordinary differential equation [20]

$$
\dot{Q}=\frac{2 \alpha\left(\lambda_{c}\right) \rho F Q^{2}}{c^{2} \rho^{2} F^{2}-Q^{2}}, \quad Q(0)=u,
$$

where $c \gg \omega_{c}$ and all quantities are assumed constant $Q=$ $\rho \omega_{c} F$ and $F$ is area of across section of the pump-compressor tubes and is constant relative to axes.

It is assumed that the passing from the end of tube through the layer to the beginning of the lift $(x=l)$ is described by the following difference equations:

$$
\begin{aligned}
Q(l+0) & =\gamma Q(l-0)+\gamma_{1}(Q(l-0)) \bar{Q}, \\
\gamma_{1}(Q(l-0)) & =-\delta_{3}\left(Q(l-0)-\delta_{2}\right)^{2}+\delta_{1},
\end{aligned}
$$

where $\gamma$ and $\delta_{1}, \delta_{2}, \delta_{3}$ are constant numbers to be found. For the sake of simplicity we suppose that the parameters $\gamma, \delta_{1}$, $\delta_{2}, \delta_{3}$ are known and it is required to reconstruct $\lambda_{c}$ involved in (19) due to $\alpha\left(\lambda_{c}\right)$.

Then some nominal trajectory $Q^{0}(x)$ and parameter $\alpha^{0}$ are chosen assuming that $k$ th iteration is already held. Let us linearize (29) among these data

$$
\begin{aligned}
\dot{Q}^{k}(x)= & A\left(Q^{k-1}, \alpha^{k-1}\right) \cdot Q^{k}(x)+B\left(Q^{k-1}, \alpha^{k-1}\right) \alpha^{k} \\
& +C\left(Q^{k-1}, \alpha^{k-1}\right)
\end{aligned}
$$

where

$$
\begin{gathered}
\frac{\partial f\left(y^{k-1}, \alpha^{k-1}\right)}{\partial y}=A\left(Q^{k-1}, \alpha^{k-1}\right) \\
=\frac{4 \alpha^{k-1} c^{2} \rho^{3} F^{3} Q^{k-1}}{\left(c^{2} \rho^{2} F^{2}-\left(Q^{k-1}\right)^{2}\right)^{2}},
\end{gathered}
$$

$$
\begin{aligned}
& \frac{\partial f\left(y^{k-1}, \alpha^{k-1}\right)}{\partial \alpha}=B\left(Q^{k-1}, \alpha^{k-1}\right) \\
& =\frac{2 \alpha^{k-1} \rho F Q^{k-1}}{c^{2} \rho^{2} F^{2}-\left(Q^{k-1}\right)^{2}}, \\
& f\left(y^{k-1}, \alpha^{k-1}\right)-\frac{\partial f\left(y^{k-1}, \alpha^{k-1}\right)}{\partial y} y^{k-1} \\
& -\frac{\partial f\left(y^{k-1}, \alpha^{k-1}\right)}{\partial \alpha} \alpha^{k-1}=C\left(Q^{k-1}, \alpha^{k-1}\right) \\
& =\frac{4 \alpha^{k-1} c^{2} \rho^{3} F^{3}\left(Q^{k-1}\right)^{2}+4 \alpha^{k-1} \rho F\left(Q^{k-1}\right)^{4}}{\left(c^{2} \rho^{2} F^{2}-\left(Q^{k-1}\right)^{2}\right)^{2}} .
\end{aligned}
$$

Note that by the help of the relations (20) and (21) the matrices $\Phi^{k-1}(x, 0), \Phi_{1}^{k-1}(x, 0), \Phi_{2}^{k-1}(x, 0)$ are calculated as follows:

$$
\begin{aligned}
& \Phi_{i}^{k-1}(x, 0)=\prod_{i=2 N-1}^{j}\left(E+A\left(Q^{k-1}\left(x_{i_{1}}\right), \alpha^{k-1}\right)\right) h, \\
& \Phi_{1 i}^{k-1}\left(x_{2 N}, 0\right) \\
& =\left(\sum_{j=N+2}^{2 N-1}\left(\prod_{i=2 N-1}^{j}\left(E+A\left(Q^{k-1}\left(x_{i_{1}}\right), \alpha^{k-1}\right)\right) h\right)\right. \\
& \left.\quad B\left(Q^{k-1}\left(x_{j-1}\right), \alpha^{k-1}\right) h\right) \\
& \quad+B\left(Q^{k-1}\left(x_{2 N-1}\right), \alpha^{k-1}\right) h,
\end{aligned}
$$


TABLE 1

\begin{tabular}{llllllll}
\hline$y_{i}^{l+0}$ & 5.5698 & 5.5732 & 5.5761 & 5.5810 & 5.5848 & 5.5852 & 5.5824 \\
\hline$y_{i}^{2 l}$ & 4.4242 & 4.4248 & 4.4254 & 4.4262 & 4.4266 & 4.4263 & 4.4251 \\
\hline
\end{tabular}

TABLE 2

\begin{tabular}{ccccccc}
\hline$\delta$ & 0.05 & 0.1 & 0.5 & 0.54 & 0.55 & 0.6 \\
\hline$\lambda$ & 0.1966 & 0.2141 & 0.2295 & 0.2298 & 0.2299 & 0.2302 \\
\hline
\end{tabular}

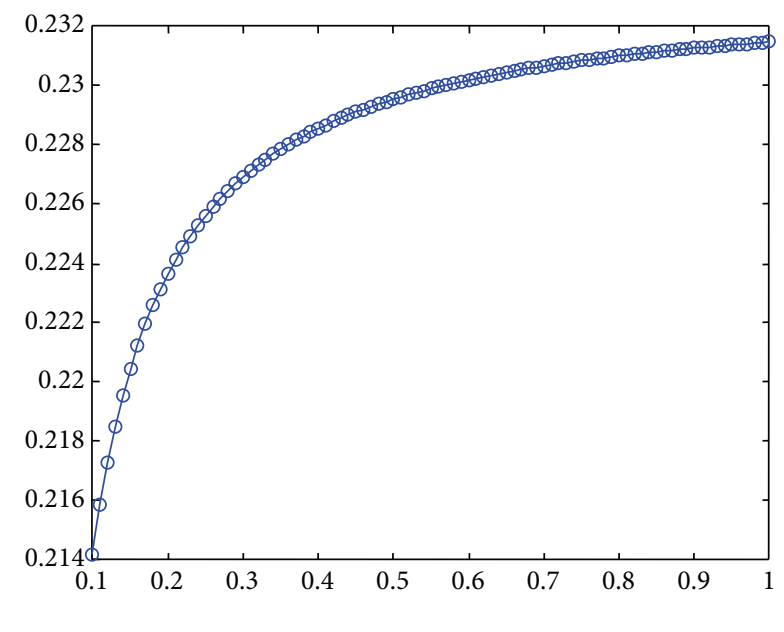

Figure 2

$$
\begin{aligned}
& \Phi_{2 i}^{k-1}\left(x_{2 N}, 0\right) \\
& =\left(\sum_{j=N+2}^{2 N-1}\left(\prod_{i=2 N-1}^{j}\left(E+A\left(Q^{k-1}\left(x_{i_{1}}\right), \alpha^{k-1}\right)\right) h\right)\right. \\
& \left.\cdot C\left(Q^{k-1}\left(x_{j-1}\right), \alpha^{k-1}\right) h\right) \\
& +C\left(Q^{k-1}\left(x_{2 N-1}\right), \alpha^{k-1}\right) h
\end{aligned}
$$

where $h$ is small enough number.

Let some statistical data be given.

Let us assume that some observation points for $y_{i}^{l+0}$ and $y_{i}^{2 l}$ are given (see Table 1 ).

We give in Table 2 the values of $\lambda$ obtained by using MATLAB by given input parameters.

As we see from Table 2 by $\delta=0.55 \lambda$ gets value 0.2299 , with error estimation $10^{-3}$.

Here is the dependence of $\lambda$ or $\delta$ (see Figure 2).

The above algorithm reaches given accuracy after 4 iterations and gives $\lambda=0.2299$.

Note that the inequality

$$
\left|\lambda_{i}-\lambda_{4}\right| \leq 10^{-15}
$$

holds for any $i>4$ that shows the stability of the proposed quasilinearization algorithm.

\section{Conflict of Interests}

The authors declare that there is no conflict of interests regarding the publication of this paper.

\section{Acknowledgment}

This work is supported by the joint grant of ANAS and SOCAR 17, 2013-2015, Baku State University “50 + 50" Grant.

\section{References}

[1] A. Aliev Fikret and N. A. Ismailov, "Inverse problem to determine the hydraulic resistance coefficient in the gas lift process," Applied and Computational Mathematics, vol. 12, no. 3, pp. 306-313, 2013.

[2] R. Gurbanov, N. Nuriyev, and R. S. Gurbanov, “Technological control and optimization problems in oil production: theory and practice," Applied and Computational Mathematics, vol. 12, no. 3, pp. 314-324, 2013.

[3] R. N. Bakhtisin and A. R. Latypov, "Estimation of the order of linear objects by experimental information," Automation and Remote Control, no. 3, pp. 108-112, 1992.

[4] Y. S. Gasimov, "On a shape design problem for one spectral functional," Journal of Inverse and Ill-Posed Problems, vol. 21, no. 5, pp. 629-637, 2013.

[5] L. Lyuing, Identification of the System. Theory for Users, Nauka, Moscow, Russia, 1991.

[6] F. A. Aliev, M. M. Mutallimov, I. M. Askerov, and I. S. Raguimov, "Asymptotic method of solution for a problem of construction of optimal gas-lift process modes," Mathematical Problems in Engineering, vol. 2010, Article ID 191053, 10 pages, 2010.

[7] A. S. Apostolyuk and V. B. Larin, "Updating of linear stationary dynamic system parameters," Applied and Computational Mathematics, vol. 10, no. 3, pp. 402-408, 2011.

[8] F. Ding, "Hierarchical multi-innovation stochastic gradient algorithm for Hammerstein nonlinear system modeling," Applied Mathematical Modelling, vol. 37, no. 4, pp. 1694-1704, 2013.

[9] F. Ding, Y. Shi, and T. Chen, "Auxiliary model-based leastsquares identification methods for Hammerstein output-error systems," Systems \& Control Letters, vol. 56, no. 5, pp. 373-380, 2007.

[10] S. I. Kabanikhin and O. I. Krivorot'Ko, "A numerical method for determining the amplitude of a wave edge in shallow water approximation," Applied and Computational Mathematics, vol. 12, no. 1, pp. 91-96, 2013.

[11] P. E. Bellman and P. E. Kalaba, Quailinearization and Nonlinear Boundary Problems, Mir, Moscow, Russia, 1968.

[12] V. E. Shamansky, Methods of Numerical Solution of BaundaryProblems in PC, Naukova Dumka Kiev, 1966.

[13] A. Brayson and X. Yu-shi, Applied Theory of Optimal Control, Mir, Moscow, Russia, 1972.

[14] D. M. Himmebblau, Applied Nonlinear Programming, Craw-Hill Book Company, New York, NY, USA, 1972.

[15] K. R. Aydazade, "Computatioonal problems in hydraulic networks," Computational Mathematics and Mathematical Physics, vol. 29, no. 2, pp. 184-193, 1989.

[16] Y. N. Andreev, Control of the Finite Dimensional Linear Objects, Nauka, Moscow, Russia, 1976. 
[17] J. R. Magnus and H. Neudecker, Matrix Differential Calculus with Applications in Statistics and Econometrics, John Wiley \& Sons, Chichester, UK, 17th edition, 1988.

[18] K. B. Petersen and M. S. Pedersen, The Matrix Cookbook, 2008, http://matrixcookbook.com.

[19] D. M. Altshul, Hidraulic Resistance, Nedra, Moscow, Russia, 1970.

[20] M. Ghanbari, S. Abbasbandy, and T. Allahviranloo, "A new approach to determine the convergence-control parameter in the application of the homotopy analysis method to systems of linear equations," Applied and Computational Mathematics, vol. 12, no. 3, pp. 355-364, 2013. 


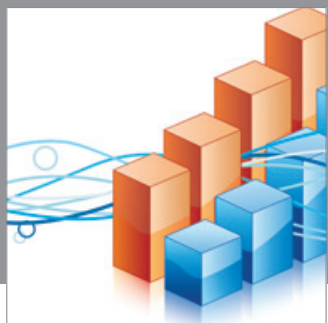

Advances in

Operations Research

mansans

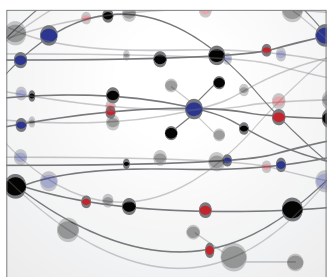

The Scientific World Journal
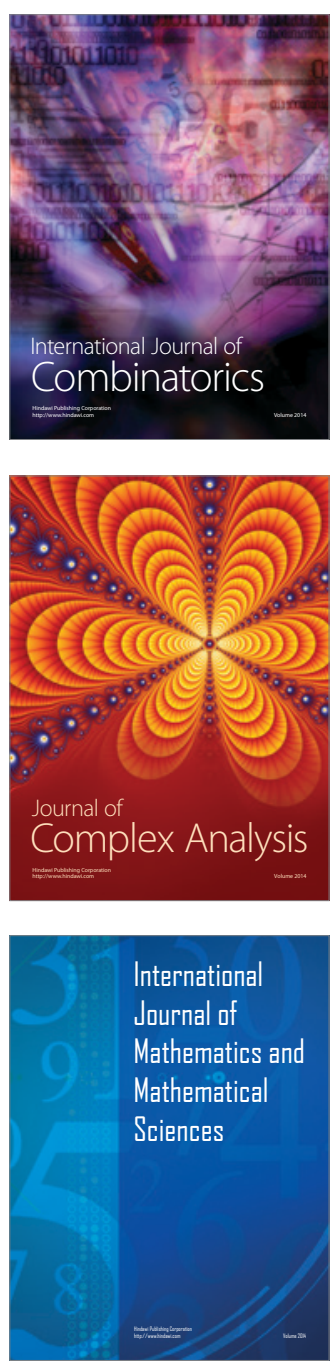
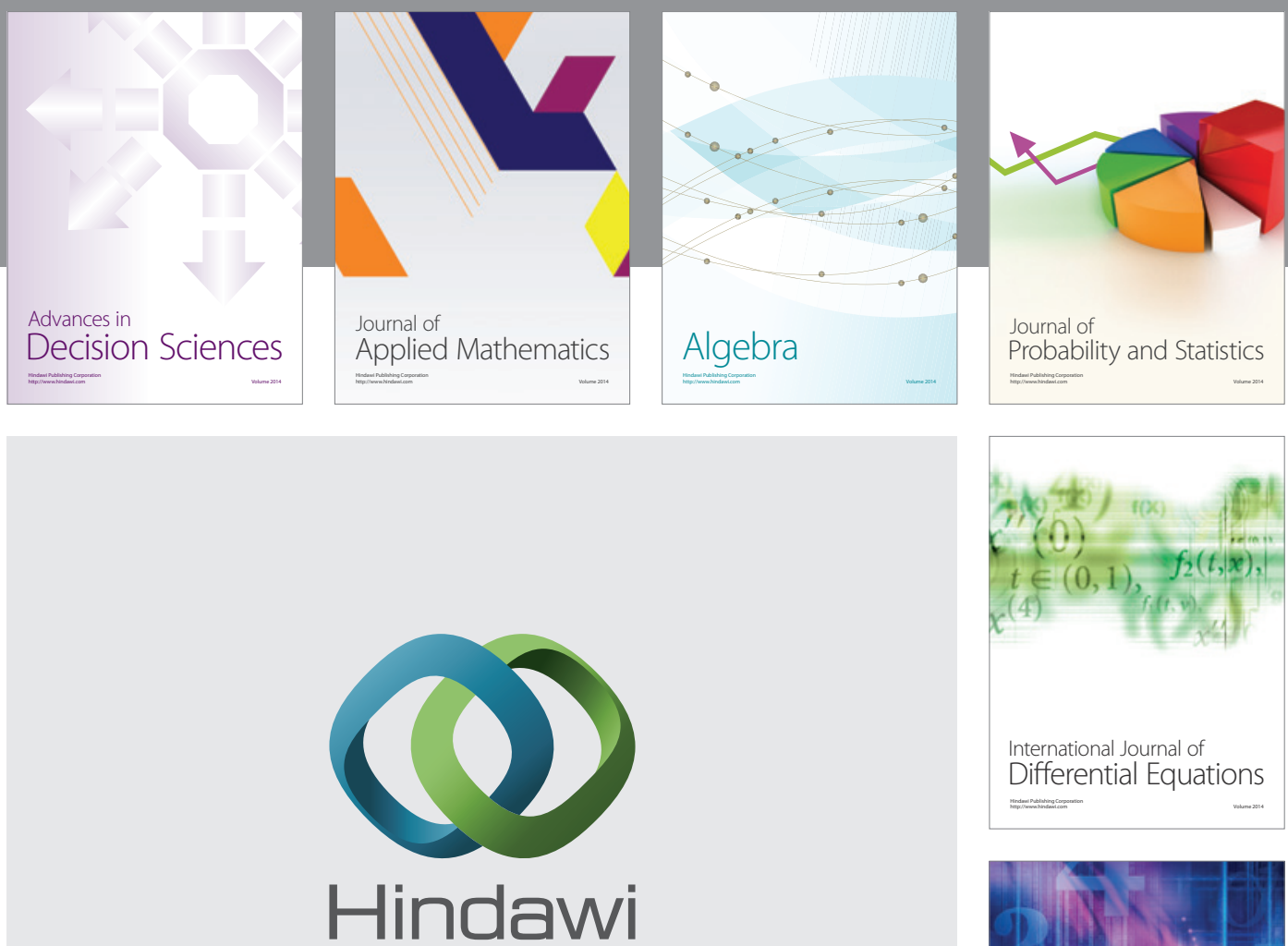

Submit your manuscripts at http://www.hindawi.com
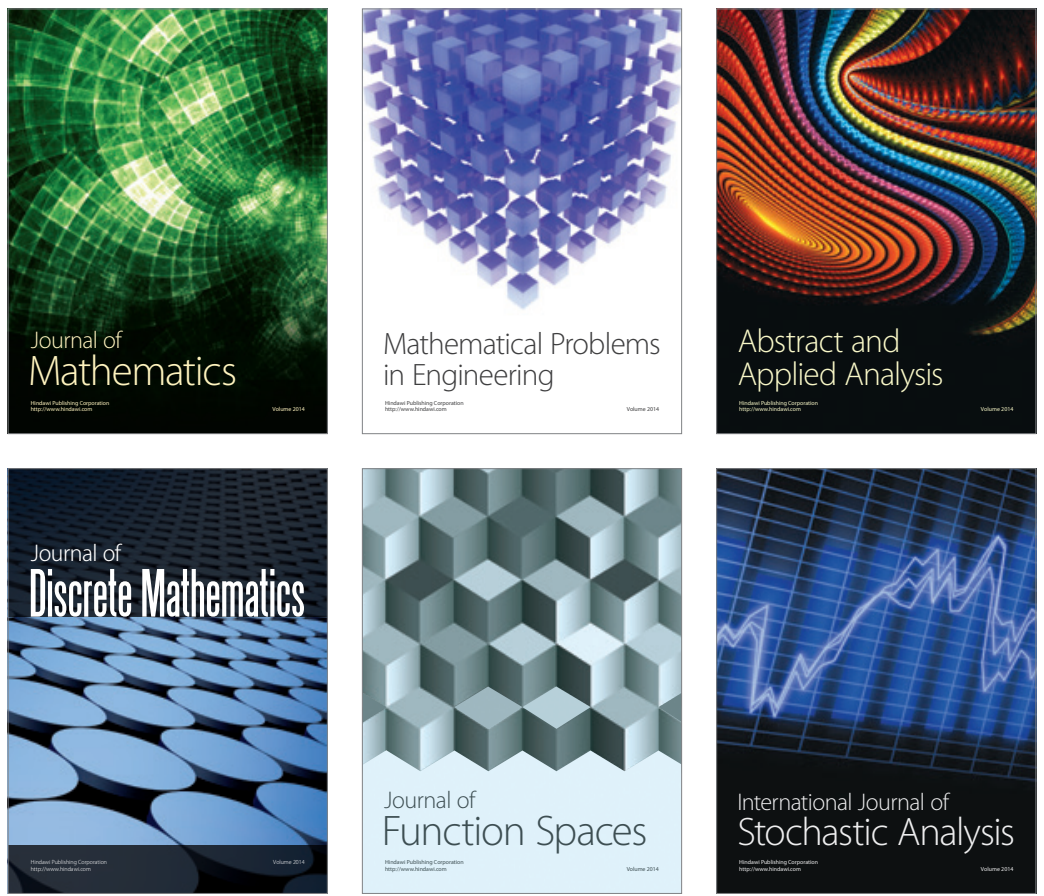

Journal of

Function Spaces

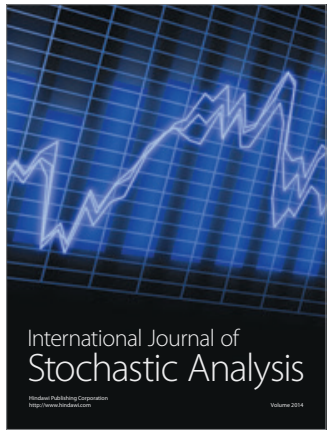

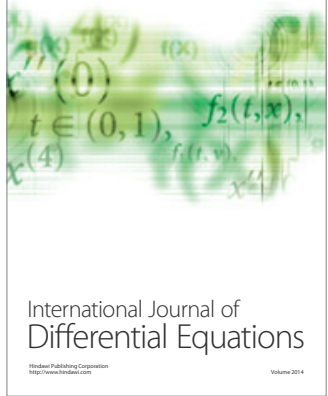
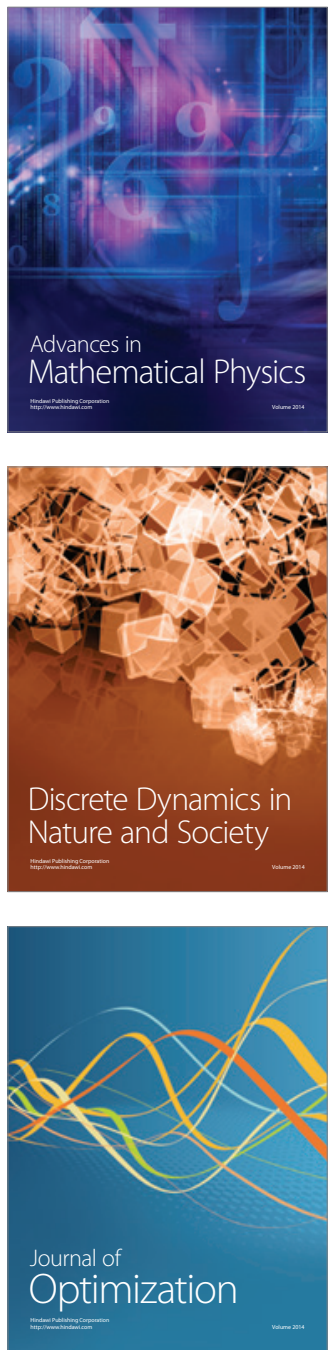